\title{
Percutaneous MR-guided focal cryoablation for recurrent prostate cancer following radiation therapy: retrospective analysis of iceball margins and outcomes
}

\author{
Christiaan G. Overduin $^{1}$ - Sjoerd F. M. Jenniskens ${ }^{1}$ • J. P. Michiel Sedelaar ${ }^{2}$ • \\ Joyce G. R. Bomers ${ }^{1}$ • Jurgen J. Fütterer ${ }^{1,3}$
}

Received: 27 December 2016 / Revised: 2 March 2017 / Accepted: 21 March 2017 / Published online: 13 April 2017

(C) The Author(s) 2017. This article is an open access publication

\begin{abstract}
Objectives To evaluate iceball margins after magnetic resonance (MR)-guided focal salvage prostate cryoablation and determine the correlation with local outcome.

Methods A retrospective review was performed on 47 patients that underwent percutaneous MR-guided focal cryoablation for biopsy-proven locally recurrent prostate cancer after primary radiotherapy. Preprocedural diagnostic and intraprocedural MR images were analysed to derive threedirectional iceball margins. Local tumour progression after cryoablation was defined as evident tumour recurrence on follow-up MRI, positive MR-guided biopsy or biochemical failure without radiological evidence of metastatic disease. Results Mean iceball margins were $8.9 \mathrm{~mm}$ (range -7.1 to 16.2), $10.1 \mathrm{~mm}$ (range 1.1-20.3) and $12.5 \mathrm{~mm}$ (range -1.5 to 22.2) in anteroposterior, left-right and craniocaudal direction respectively. Iceball margins were significantly smaller for tumours that were larger $(P=.008)$ or located in the posterior gland $(P=.047)$. Significantly improved local progression-free survival at 1 year post focal cryoablation was seen between patients with iceball margin $>10 \mathrm{~mm}$ (100\%), 5-10 mm (84\%) and <5 mm (15\%) $(P<.001)$.
\end{abstract}

Christiaan G. Overduin

kristian.overduin@radboudumc.nl

1 Department of Radiology and Nuclear Medicine, Radboud University Medical Center, P.O. Box 9101 (767), 6500 HB Nijmegen, The Netherlands

2 Department of Urology, Radboud University Medical Center, Nijmegen, The Netherlands

3 MIRA Institute for Biomedical Engineering and Technical Medicine, University of Twente, Enschede, The Netherlands
Conclusions Iceball margins appear to correlate with local outcome following MR-guided focal salvage prostate cryoablation. Our initial data suggest that freezing should be applied at minimum $5 \mathrm{~mm}$ beyond the border of an MRvisible recurrent prostate tumour for successful ablation, with a wider margin appearing desirable.

Key points

- Shortest iceball margin most often occurred in anteroposterior direction

- Margins were smaller in tumours that were larger or posteriorly located

- Minimum iceball margin was a predictor of early local tumour progression

- A minimum 5-mm margin seems required for effective cryoablation of recurrent $\mathrm{PCa}$

Keywords Ablation margin · Cryoablation · MRI · Prostate cancer $\cdot$ Local recurrence

\section{Introduction}

Prostate cancer $(\mathrm{PCa})$ recurrence following radiotherapy (RT) is not uncommon, with an estimated $10-60 \%$ of patients experiencing biochemical failure within 5-10 years after treatment $[1,2]$. There has been no consensus on optimal management of this patient group with a majority of patients receiving androgen deprivation therapy (ADT) [3]. Treatment options with curative intent are limited and include salvage radical prostatectomy (RP), brachytherapy, high-intensity focused ultrasound and cryoablation [4].

Salvage RP is generally regarded as a challenging procedure to perform and has been associated with high complication risks [5]. Whole-gland salvage cryoablation has emerged as an accepted treatment alternative, with acceptable 
oncological outcomes and side effects [6, 7]. However, complications such as erectile dysfunction $(72-100 \%)$, incontinence $(2.6-73 \%)$ and rectal fistula $(0-3.4 \%)$ may still exist $[8,9]$. In an attempt to reduce treatment morbidity, several studies have been exploring the concept of partial cryoablation for biopsy-proven unilateral recurrences [9-11]. At the same time, advancements in prostate magnetic resonance (MR) imaging have enabled the accurate detection and localization of locally recurrent $\mathrm{PCa}$ after previous irradiation $[12,13]$. Additionally, MRI can also be used to intraoperatively guide cryoablation procedures [14, 15], opening new possibilities for focal salvage approaches.

In a recent study the feasibility of MR-guided focal cryoablation was shown in ten patients with locally recurrent PCa after primary RT [16]. Although initial short-term results were promising, three patients developed local tumour recurrence at the site of ablation within 6-12 months after treatment and the authors concluded that these tumours were most probably undertreated. Findings from in vitro and in vivo animal studies on renal cryoablation have shown that lethal temperatures may be achieved at approximately 5-6 $\mathrm{mm}$ within the edge of the iceball $[17,18]$. For prostate cryoablation, some authors have suggested freezing to be applied $2-3 \mathrm{~mm}$ beyond the tumour boundary to reach an end temperature of $-20{ }^{\circ} \mathrm{C}$, which has been associated with tissue necrosis [19]. However, limited clinical data is presently available verifying these recommendations with patient outcomes and a consensus on the margin needed for effective cryoablation of recurrent PCa has not been established. The purpose of the present study was to evaluate iceball margins after MR-guided focal salvage prostate cryoablation and determine the correlation with local outcome.

\section{Materials and methods}

In July 2016, a total of 61 consecutive patients were identified that underwent MR-guided focal cryoablation for locally recurrent PCa at our institution between May 2011 and July 2015 and were at least 1 year after treatment. Ten patients were excluded because they had undergone previous salvage treatment. Additionally, three patients who underwent radical prostatectomy as primary treatment and one patient who was treated in two separate sessions were excluded. A total of 47 patients (median age 66 years, age range 52-79) underwent MR-guided focal cryoablation for local PCa recurrence after primary RT and were included in this retrospective study. Informed consent to use anonymized data for analysis was obtained in all patients.

Patients were eligible for focal cryoablation on the basis of biopsy-proven MRI-visible local PCa recurrence without radiologic evidence of metastatic disease. The final decision to perform cryoablation was made at the multidisciplinary board meeting. Focal cryoablation was performed at a median of 5 years (range 1-18) after primary RT. Prior to treatment, all patients underwent 3-T multi-parametric MR imaging (mpMRI) with a pelvic phased-array coil $(n=43)$ or endorectal coil $(n=4)$, consisting of T2-weighted (T2w), diffusion-weighted (DWI) and dynamic contrast-enhanced (DCE) imaging, to localize the recurrence and measure visible tumour volume as well as an abdominal and pelvic staging MRI to exclude nodal or bone marrow metastases. There were no restrictions related to pretreatment prostate specific antigen (PSA) level, Gleason score, prior history of ADT, tumour volume or local extent. The National Comprehensive Cancer Network (NCCN) guidelines were used to categorize patients into low-to-intermediate and high-risk groups [20]. High-risk status was assigned when any of the following criteria were met: cancer stage $\geq \mathrm{T} 3 \mathrm{a}$, Gleason $\geq 8$ or PSA $\geq 20 \mathrm{ng} / \mathrm{ml}$. A summary of patient demographics is included in Table 1.

\section{Cryoablation procedures}

Patients were treated under general anaesthesia in a 1.5-T or 3T MR system (Magnetom Avanto or Skyra, Siemens, Erlangen, Germany). All procedures were performed by one of two interventional radiologists (J.F. or S.J.) each with more than 6 years of experience in prostate interventions. A urethral warming catheter and rectal balloon were inserted to protect the urethra and rectal wall. A transperineal approach was used to place multiple MR-compatible cryoprobes (MRI IceSeed or IceRod, Galil Medical, Yokneam, Israel) centrally in the target region under real-time MR image guidance. Type, number and position of cryoprobes were chosen at the discretion of the performing physician. Cryoablation was performed using an MR-compatible cryoablation device (MRI-SeedNet; Galil Medical, Yokneam, Israel). Two 10-min freezing cycles separated by 2-min passive and 1-min active thaw were applied under continuous T1-weighted gradient echo MR monitoring $[21,22]$. The intraprocedural images were used to modulate the size and shape of the iceball by regulating the gas flow to each individual cryoprobe so as to cover the entire tumour while avoiding contact between the iceball and rectal wall. Upon completion of the ablation, all cryoprobes were thawed and removed. All intraprocedural complications were documented. Postprocedural complication data, functional outcomes and quality-of-life metrics were not part of the present work and will be reported elsewhere.

\section{Image review and follow-up}

All preprocedural and intraprocedural images were retrospectively reviewed by one prostate interventional radiologist (S.J.) using medical image visualization software (MeVisLab, Fraunhofer, Bremen, Germany). Treated tumours were annotated on the pretreatment diagnostic mpMRI using information 
Table 1 Patient demographic data $(N=47)$

\begin{tabular}{ll}
\hline & Median (range) or $n(\%)$ \\
\hline Age (years) & $66(52-79)$ \\
PSA level (ng/ml) & $4.9(0.7-31.0)$ \\
Form of primary RT & $29(62)$ \\
$\quad$ EBRT & $17(36)$ \\
Brachytherapy & $1(2)$ \\
EBRT + brachytherapy & $5(1-18)$ \\
Time to recurrence since primary RT (years) & \\
Prior ADT use & $17(36)$ \\
$\quad$ Yes & $30(64)$ \\
No & \\
Gleason score of recurrence & $7(15)$ \\
$\quad \leq 6$ & $17(36)$ \\
7 & $16(34)$ \\
$\quad \geq 8$ & $7(15)$ \\
Unknown or undeterminable & \\
Clinical stage & $35(69)$ \\
T2a-c & $12(31)$ \\
T3b & $18(38)$ \\
NCCN risk category & $29(62)$ \\
Low-to-intermediate & $23.5(6.6-64.2)$ \\
High & \\
Prostate volume (ml) & $11(23)$ \\
Tramour localization & \\
Peripheral zone and/or seminal vesicles & \\
Tumour volume (ml) & \\
\hline & \\
\hline &
\end{tabular}

$E B R T$ external beam radiotherapy, $A D T$ androgen-deprivation therapy, $N C C N$ National Comprehensive Cancer Network

from all available MR sequences to delineate the tumour boundary. Tumour locations were classified as anterior when more than 50\% located in the ventral half of the prostate or posterior when located more than $50 \%$ in the dorsal half of the prostate.

Intraprocedural MR images obtained at the end of the second freeze cycle were used to annotate each corresponding iceball. MeVisLab software was used register each annotated iceball to the centre of the corresponding annotated tumour. Maximum tumour and corresponding iceball sizes were derived from the annotated regions in anteroposterior, left-right and craniocaudal direction as well as total tumour and iceball volumes. The iceball margin was then defined as the margin calculated by subtracting the iceball's radius from the corresponding lesion radius in each direction (Fig. 1). The minimum iceball margin was defined as the shortest margin in each patient. Undertreated tumours were defined by a negative margin between the iceball and tumour. Finally, the position of the iceball relative to the tumour was recorded: a well-centred iceball covered the entire tumour in the axial plane at the centre of the lesion.
Postprocedural follow-up (median 24 months, range 3-42) consisted of regular urology visits with PSA monitoring at 1 , $3,6,9$ and 12 months after treatment, followed by 3 - to 6-month intervals. In addition, all patients underwent follow-up prostate mpMRI at 3, 6 and 12 months after treatment. All follow-up images were reviewed by one of two prostate interventional radiologists (J.F. or S.J.). Any finding suspected of indicating residual or recurrent tumour was subsequently sampled by targeted in-bore MR-guided biopsy. Furthermore, all patients received targeted in-bore MR-guided biopsy directed at the ablation zone at 12 months post-treatment from October 2013 onwards. Local tumour progression after focal cryoablation was defined as evident tumour recurrence on follow-up MRI, positive MR-guided biopsy or biochemical failure according to Phoenix criteria of PSA nadir $+2 \mathrm{ng} / \mathrm{ml}$ [23] without radiological evidence of metastatic disease.

\section{Statistical analysis}

Statistics were performed using SPSS (version 20.0). Data are reported as mean \pm standard deviation unless indicated otherwise. Baseline parameters were compared between cases of local control and tumour progression using independent samples $t$ test for normally distributed and Mann-Whitney $U$ test for non-normally distributed data. Chi-square analysis was used for discrete data. Independent samples $t$ test was used to compare iceball margins by tumour volume and location. Survival analysis was performed using the Kaplan-Meier method. Log-rank test was used to assess differences between survival curves. Cox proportional hazard univariable and multivariable models were used to assess pretreatment risk status, prior ADT use and tumour volume as potential confounders and determine whether iceball margins were independently associated with local progression. Potential prognostic factors with a $P$ value less than 0.10 on univariable analysis were included in the multivariable model. Outcomes were reported as hazard ratios (HR) with $95 \%$ confidence intervals (CI) to demonstrate level of precision. $P$ values less than 0.05 were considered statistically significant.

\section{Results}

A total of 47 cryoablation procedures were successfully performed in 47 patients. A median of 3 cryoprobes (range 2-6) were used per patient. The median iceball volume was $27.7 \mathrm{ml}$ (range 6.0-82.5). There were no intraprocedural complications. The median PSA nadir after focal prostate cryoablation was $0.6 \mathrm{ng} / \mathrm{ml}$ (range $<0.02-40.0$ ) and was reached at a median of 3 months (range 1-24) after treatment.

On follow-up, 24 of 47 patients (51\%) were locally controlled. Local tumour progression occurred in 23 patients 

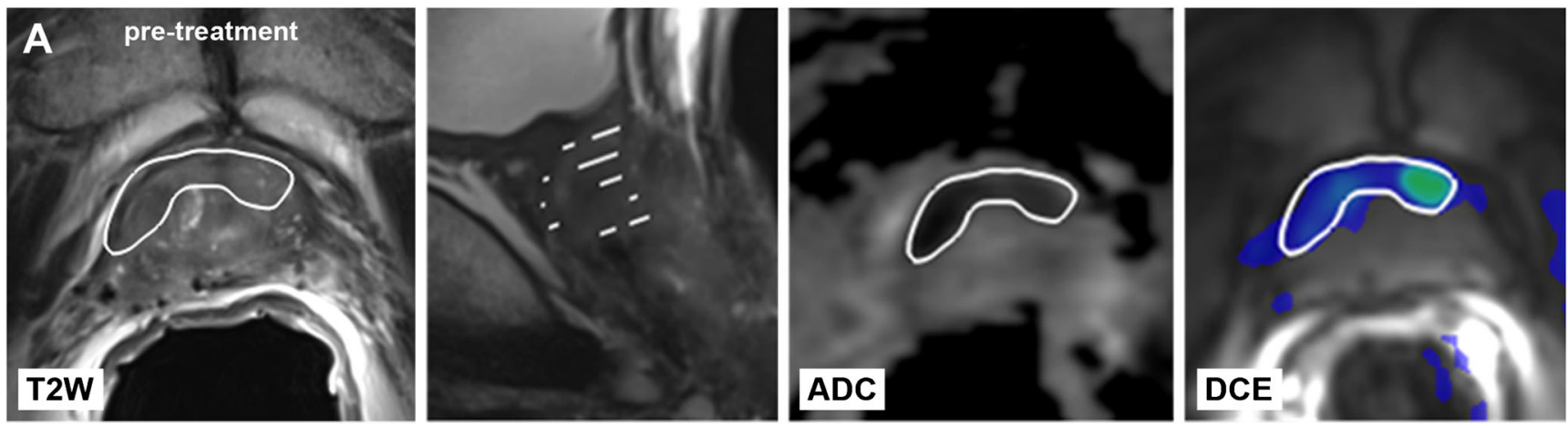
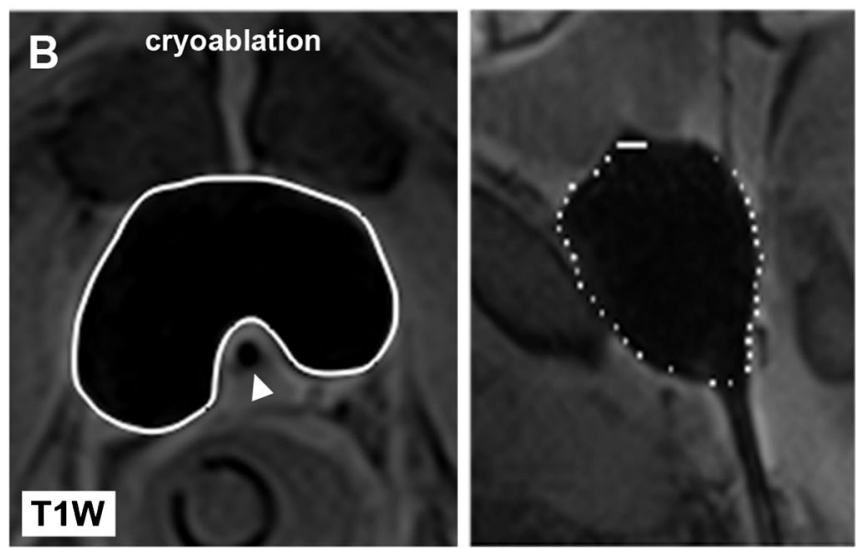

Fig. 1 Annotation of a tumour (outlined in white) on pretreatment axial and sagittal T2-weighted, ADC and DCE prostate MR images and b corresponding iceball (outlined in white) at the end of the second freeze cycle on axial and sagittal intraprocedural T1-weighted images, with

(49\%), of whom 14 had a positive MR-guided prostate biopsy, seven experienced PSA failure without radiological evidence of distant disease and two patients had evident tumour recurrence on follow-up MRI but were spared targeted biopsy because of detection of nodal or bone metastases on the same scan. The median time between treatment and local tumour progression was 12 months (range 3-42). After multidisciplinary consensus seven patients with local tumour progression received repeat treatment with MR-guided cryoablation. Ten out of the 47 patients (21\%) showed metastatic disease progression, of whom six were locally controlled and four patients also had evidence of local tumour progression.

The distribution of baseline parameters between cases of local control and tumour progression is shown in Table 2. A statistically significant difference was found in prior history of ADT administration between the stable and local progression group ( $5 / 24$ vs. $11 / 23 ; P=.025)$. No difference was seen for pretreatment PSA level, Gleason score and clinical stage.

\section{Iceball margins}

The mean iceball margins for focal cryoablation were $8.9 \mathrm{~mm}$ (range -7.1 to 16.2 ), $10.1 \mathrm{~mm}$ (range 1.1-20.3) and $12.5 \mathrm{~mm}$ (range -1.5 to 22.2 ) in anteroposterior, left-right and craniocaudal directions respectively. The mean minimum

\section{C}

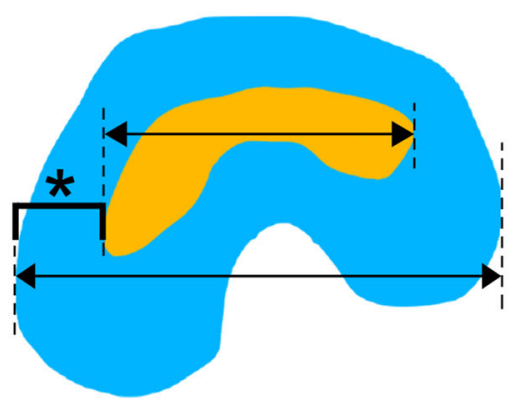

urethral warming catheter in situ (arrowhead). c The iceball margin (asterisk) was determined by subtracting the radii of the tumour (yellow) and iceball (blue) along each direction

iceball margin per patient was $7.5 \mathrm{~mm}$ (range -7.1 to 14.2 ), with the shortest margin most often occurring in anteroposterior direction (49\%). An iceball margin greater than $5 \mathrm{~mm}$ in each direction was achieved in $34 / 47(72 \%)$ of patients. Negative margins occurred in three cases $(6 \%)$. Iceballs were well centred in all but one patient. The one off-centre iceball occurred in a large tumour involving the peripheral zone and seminal vesicles. The iceball was found to be off-centre on retrospective review of the intraprocedural images because the insertion angle of the cryoprobes was incorrectly aligned with the tumour's geometry, causing the iceball to be off-centre at the prostatic base and seminal vesicles.

When stratified by tumour volume and location, the average minimum iceball margin was significantly smaller for tumours larger than $1 \mathrm{ml}$ compared to tumours $1 \mathrm{ml}$ or smaller $(5.8 \pm 4.8$ vs. $9.2 \pm 3.4 \mathrm{~mm} ; P=.008)$ (Fig. 2$)$ and for posterior versus anterior tumours $(6.8 \pm 4.7$ vs. $9.8 \pm 2.7 \mathrm{~mm} ; P=.047)$ (Fig. 3).

On Kaplan-Meier analysis, significantly improved local tumour progression-free survival at 1 year after focal cryoablation was seen between patients with minimum iceball margin greater than $10 \mathrm{~mm}(100 \%), 5-10 \mathrm{~mm}(84 \%)$ and less than $5 \mathrm{~mm}(15 \%)(P<.001)$ (Fig. 4). Representative cases with and without local tumour progression after focal salvage 
Table 2 Comparison of baseline parameters

\begin{tabular}{lccc}
\hline & Local control $(N=24)$ & Local tumour progression $(N=23)$ & $P$ value \\
\hline PSA level & $6.8(0.7-31.0)$ & $7.1(1.1-21.0)$ & .566 \\
$\begin{array}{l}\text { Gleason score } \\
\leq 7\end{array}$ & $14(58)$ & $10(43)$ & .604 \\
$>7$ & $8(33)$ & $8(35)$ & \\
Unknown & $2(8)$ & $5(22)$ & .154 \\
Clinical stage & & & \\
T2a-c & $20(83)$ & $15(65)$ & .025 \\
T3b & $4(17)$ & $8(35)$ & \\
Prior ADT use & & $11(48)$ & \\
Yes & $5(21)$ & $12(52)$ & \\
No & $19(79)$ &
\end{tabular}

Values in parentheses are range or percentages cryoablation are shown in Figs. 5 and 6 respectively. Univariable and multivariable Cox regression analyses are shown in Table 3. Minimum iceball margin $(P<.001)$, tumour volume $(P=.005)$ and prior ADT use $(P=.014)$ showed significant association with local outcome on univariable analysis. On multivariable analysis, minimum iceball margin $(P=.004)$ and prior ADT use $(P=.008)$ remained independent predictors of local tumour progression.

\section{Discussion}

The concept of focal therapy is to treat a tumour focus with an adequate margin while sparing as much surrounding healthy tissue as possible [24]. In cryoablation, the lethal temperature threshold is generally regarded to lie between $-20{ }^{\circ} \mathrm{C}$ to $-40{ }^{\circ} \mathrm{C}$, depending on freezing rate, freeze duration and the repetition of freeze-thaw cycles [25]. With lethal temperatures residing several millimetres behind the leading edge of the freezing process [26], it is imperative to achieve a definitive margin with sufficient ice extending beyond the tumour border to ensure effective tissue necrosis throughout the entire target region. For renal cryoablation, studies have

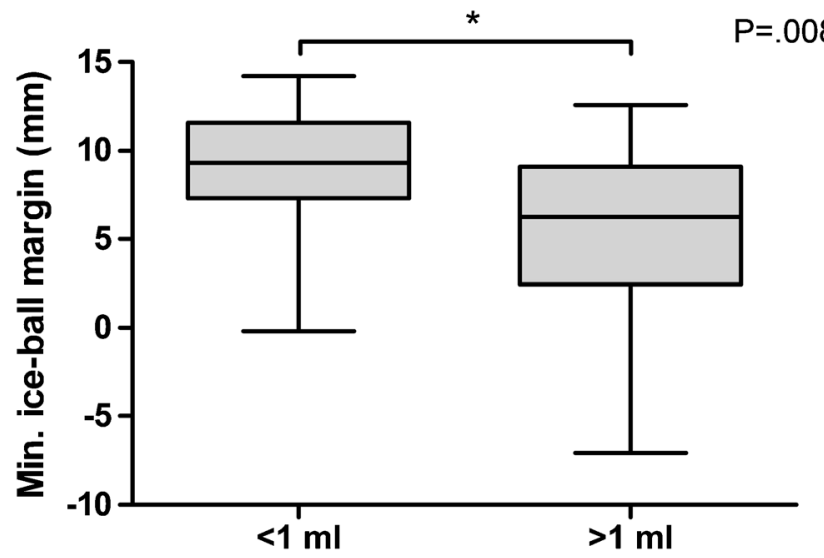

Fig. 2 Minimum iceball margin stratified by tumour volume recommended freezing to be applied for approximately 5 $6 \mathrm{~mm}$ beyond the tumour border to achieve suitably cold end temperatures [18, 27, 28]. In contrast, another study suggested that for liver cryoablation with a single cryoprobe a margin up to $1 \mathrm{~cm}$ might not be sufficient [29], but the synergistic effect of multiple cryoprobes was not studied [26, 30]. Furthermore, it is unclear how findings from other areas of application, i.e. renal or liver cryoablation, apply to the prostate salvage setting. Adding to this, uncertainty exists in predicting tumour borders from MRI with respect to the true histological tumour boundary. For primary $\mathrm{PCa}$, one study has recently shown that imaging-derived tumour boundaries from prostate mpMRI tend to underestimate the histologically determined tumour volume at prostatectomy [31]. The authors concluded that a treatment margin extending $9 \mathrm{~mm}$ around an MRI visible tumour would be required to ensure treatment of the complete tumour volume during focal ablative therapy [32]. A similar study investigating the accuracy of MR-based prostate tumour delineations for radiotherapy planning recommended a margin of $5 \mathrm{~mm}$ to achieve adequate coverage [33]. In the present work, MR imaging-derived iceball margins were correlated with local outcome after focal salvage prostate cryoablation. A high rate of early local

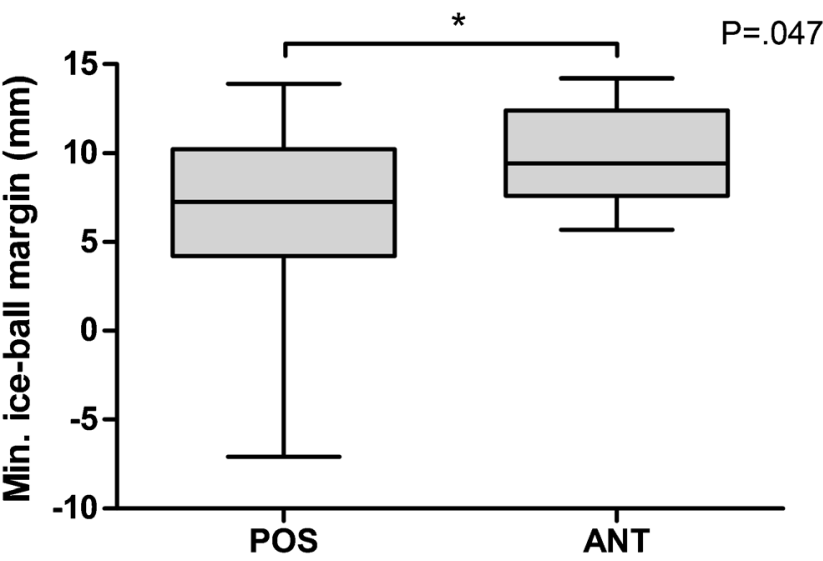

Fig. 3 Minimum iceball margin by anterior or posterior tumour locations 
$\mathrm{P}<.001$

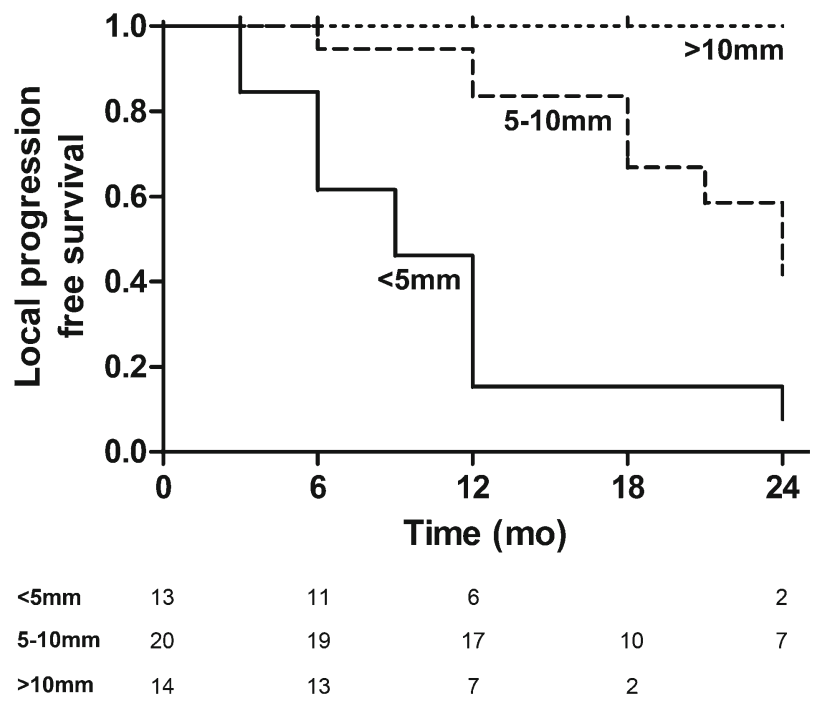

Fig. 4 Local progression-free survival stratified by minimum iceball margin $<5 \mathrm{~mm}, 5-10 \mathrm{~mm}$ and $>10 \mathrm{~mm}$, with corresponding numbers at risk tumour progression ( $85 \%$ at 1 year) was noted when the iceball margin around an MRI-visible recurrent prostate tumour were less than $5 \mathrm{~mm}$. These initial clinical data suggest that a minimum 5-mm margin would be required for effective focal salvage prostate cryoablation, with a wider margin appearing desirable.

A notable finding was that the iceball margins varied significantly with tumour location. In the posterior area of the prostate it can be particularly challenging to achieve sufficient ice coverage extending beyond the tumour boundary because of its close proximity to the rectum. During ablation, adjacency of the iceball to the rectal wall was actively monitored in the intraoperative MR images and freeze intensity was modulated accordingly to avoid unwanted damage to the rectal wall. Also, the rectum was actively warmed with warm saline using a rectal balloon, which may explain the lower iceball margins achieved in posterior tumours. For these tumour localizations, critical preoperative assessment of the ability to achieve adequate ablation margins should be performed in each individual case. Additionally, displacement of the rectum using hydrodissection may be considered to obtain increased spacing between the prostate and rectal wall and allow for a wider ablation margin.
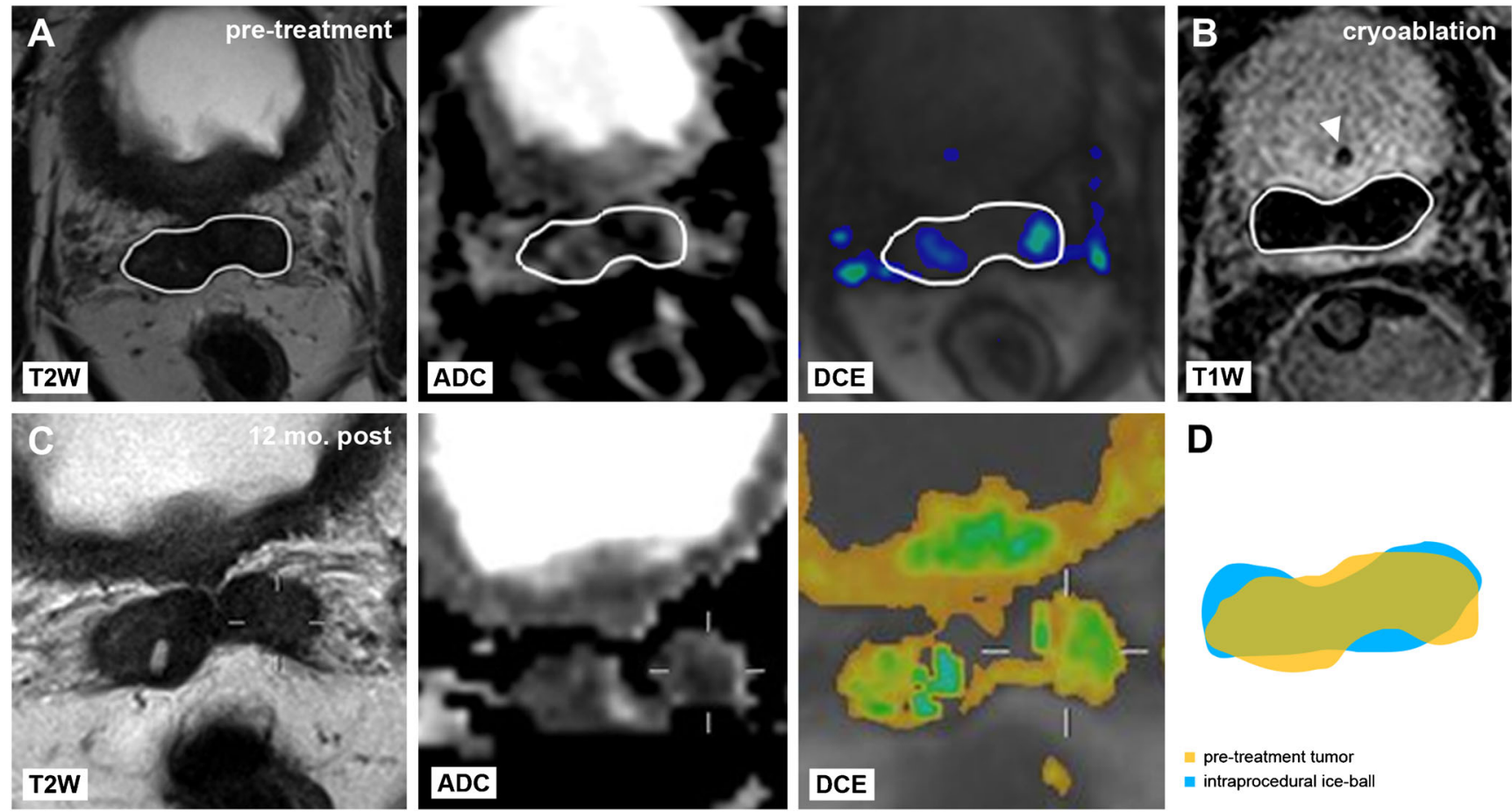

D

shows the final frozen zone at the end of the second freeze cycle, with annotated iceball boundary overlaid (white outline). Urethral warming catheter is indicated (arrowhead). c 12-month follow-up MRI shows hypointense signal on axial T2-weighted images with diffusion restriction and focal enhancement in the seminal vesicles. Local recurrence after cryoablation was confirmed on targeted in-bore MR-guided biopsy. d Retrospective review of this case showed a minimum iceball margin of $-7.1 \mathrm{~mm}$, with insufficient coverage in anteroposterior direction 

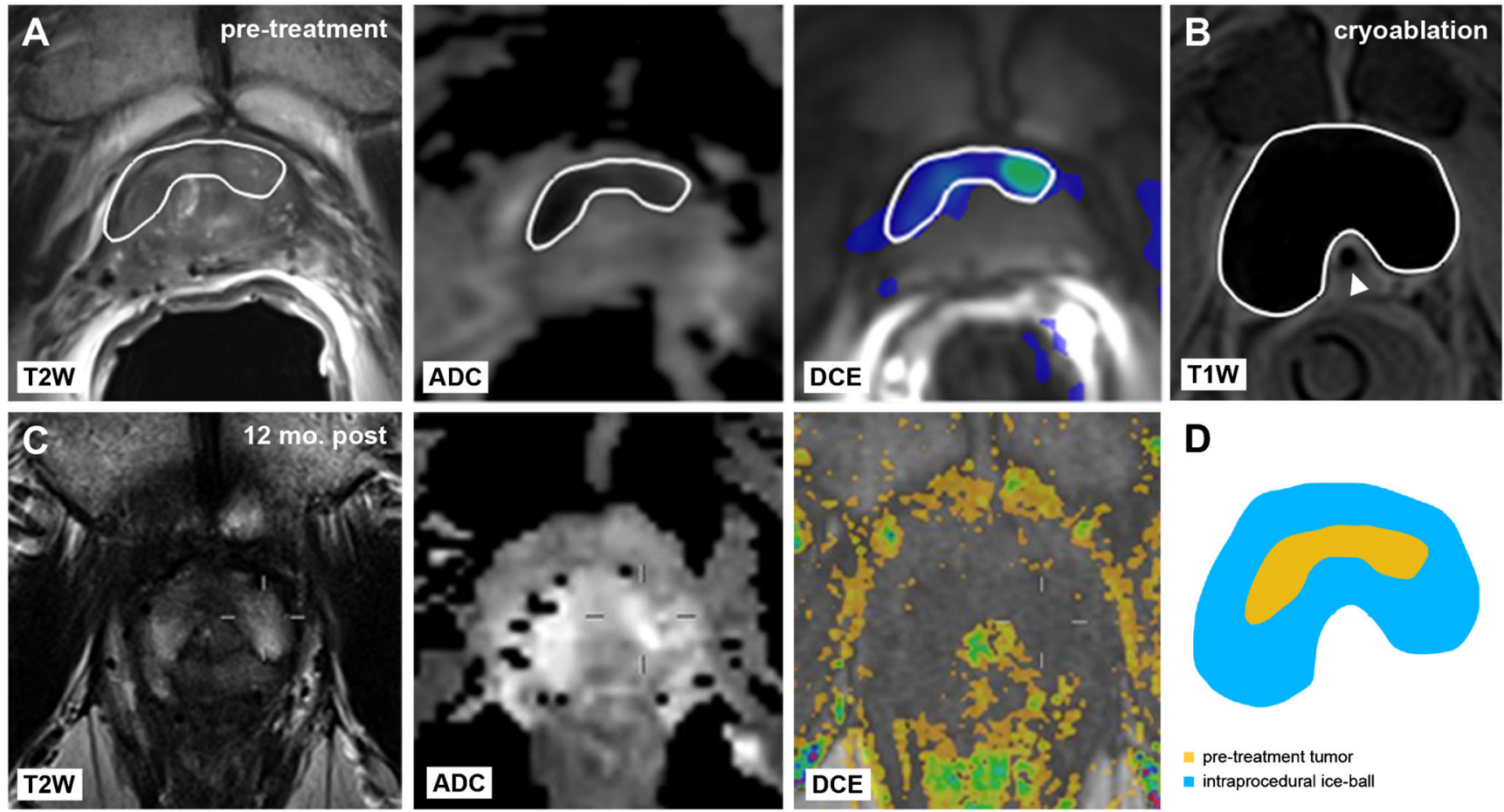

D

Fig. 6 Representative case of local tumour control. a Pretreatment axial T2-weighted, ADC and DCE images demonstrate a histologically confirmed local PCa recurrence with diffusion restriction and focal enhancement in the ventral transition zone (white outline) after primary brachytherapy. b Intraprocedural axial T1-weighted image shows the final frozen zone (white outline) at the end of the second freeze cycle, with urethral warming catheter indicated (arrowhead). c 12-month

follow-up MRI shows hyperintense signal on axial T2w images without diffusion restriction at the ventral transition zone. DCE images show a non-perfused area at the same location that coincides with the treated area. Targeted in-bore MR-guided biopsy directed at the ablation area showed no evidence of malignancy. d The retrospective review of this case showed adequate coverage in all directions, with the minimum iceball margin being $9.4 \mathrm{~mm}$

There also was a significant difference in the average iceball margin depending on tumour volume. However, substantial overlap can be seen between the margins achieved in the larger $(>1 \mathrm{ml})$ and smaller $(<1 \mathrm{ml})$ lesions and successful ablations were achieved in tumours up to $3.3 \mathrm{ml}$ in volume when a $5-\mathrm{mm}$ margin was achieved. Likewise, local tumour progression occurred after ablation of lesions as small as $0.3 \mathrm{ml}$ when the margin was insufficient in one direction. Together, these findings suggest that even though it is more difficult to achieve wide ablation margins in larger tumours, successful ablation can be achieved. In addition, in small tumours, the need for an adequate iceball margin remains important.

Of baseline parameters, prior history of ADT use was significantly different between cases of local control and tumour progression and demonstrated significant association with local tumour progression on multivariate analysis as well. Although large randomized studies have shown ADT to improve cancer-specific and overall survival when given neoadjuvantly to primary radiation $[34,35]$, the exact role of ADT before salvage treatment has not been well defined. An early study has investigated the effect of the combination of androgen deprivation with salvage surgery in patients with radiorecurrent prostate cancer and suggested that patients in whom an initial trial of ADT failed were poor candidates for salvage prostatectomy [36]. Similarly, for whole-gland salvage prostate cryoablation one study has recently shown that patients receiving ADT before salvage cryoablation had worse 5 -year biochemical progression-free survival [37]. One explanation is that the initiation of ADT before salvage cryoablation may have concealed rising PSA levels, masking
Table 3 Cox regression analysis of predictors of local progression

\begin{tabular}{lllll}
\hline & $\begin{array}{l}\text { Univariable } \\
\text { HR }(95 \% \mathrm{CI})\end{array}$ & $P$ value & $\begin{array}{l}\text { Multivariable } \\
\text { HR }(95 \% \mathrm{CI})\end{array}$ & $P$ value \\
\hline Min. iceball margin & $0.85(0.78-0.93)$ & $<.001$ & $0.85(0.77-0.95)$ & .004 \\
Tumour volume & $1.25(1.07-1.45)$ & .005 & $1.08(0.91-1.28)$ & .40 \\
Prior ADT use & $0.35(0.15-0.81)$ & .014 & $0.30(0.12-0.73)$ & .008 \\
NCCN risk category & $1.67(0.70-3.97)$ & .25 & - & \\
\hline
\end{tabular}


micrometastatic disease progression. Consequently, at the time of salvage cryoablation patients with systemic disease may be unintentionally subjected to local salvage treatment.

Possibly, the latter is corroborated by the substantial proportion of patients diagnosed with metastatic disease progression during follow-up of the present study (21\%). All but two of these instances occurred within the first 12 months after focal salvage treatment and metastatic work-up may have been false negative in these patients, with current imaging techniques having limited sensitivity [38]. Improved metastatic screening using new contrast agents such as iron nanoparticles-enhanced MR lymphography [38, 39] or gallium-68-PSMA PET-CT [40] to specifically evaluate lymph node status may be imperative to better select suitable candidates for local salvage treatment.

The most important limitation of our study was that iceball margins were only calculated in three directions and assumed the iceball to be well centred with respect to the tumour to register the annotated regions. In some patients, margins may not necessarily have been symmetrical and there could be a sufficient margin on one side but an insufficient margin on the other side when a tumour was not centrally located within the ablation zone. Ideally, all pretreatment images would be exactly matched to the intraprocedural data to provide an improved assessment of the 3D ablation margin. In our experience, however, direct registration of the image sets will not necessarily be accurate because of prostate deformation from cryoprobe insertion or movement of the entire gland due to the rectal balloon, and adequate methods to address these errors are needed to provide a more detailed characterization of the true ablation margin in future studies.

Other limitations to this work include its retrospective nature and short- to intermediate-term follow-up. Also, there is currently no universally accepted definition of tumour recurrence following local prostate salvage treatments. The Phoenix definition is the most commonly reported criterion in this setting and in one study was found to more accurately predict local cancer recurrence following prostate cryoablation than American Society for Therapeutic Radiotherapy and Oncology (ASTRO) criteria [41]. Imaging follow-up of the prostate after focal therapy using mpMRI has also been increasingly applied, with functional techniques showing promise to detect local $\mathrm{PCa}$ recurrence after therapy [42]. In this study, both biochemical as well as mpMRI and biopsy findings were integrated into a combined definition of local tumour progression. Ultimately, a standardized definition would need to be identified to facilitate direct comparison of studies evaluating prostate focal ablation techniques. Finally, heterogeneity can be seen in our patient cohort with respect to presalvage PSA levels, Gleason scores and clinical stage. Our findings require validation on multivariable analysis in more patients and with longer follow-up to come to definitive conclusions.
In conclusion, iceball margins appear correlated with local outcome following MR-guided focal salvage prostate cryoablation. Our initial data suggest that a minimum margin of $5 \mathrm{~mm}$ should be achieved around an MR-visible recurrent tumour for successful ablation, with a wider margin being desirable. Adequate margins may be more difficult to achieve in tumours that are larger and located posteriorly, which may be an important factor to consider when selecting candidates for focal salvage treatment after previous RT.

\section{Compliance with ethical standards}

Guarantor The scientific guarantor of this publication is Dr. Jurgen J. Fütterer.

Conflict of interest The authors of this manuscript declare no relationships with any companies whose products or services may be related to the subject matter of the article.

Funding The authors state that this work has not received any funding.

Statistics and biometry Dr. N. Peer, Health Evidence, Radboudumc kindly provided statistical advice for this manuscript.

Informed consent Written informed consent was obtained from all subjects (patients) in this study.

Ethical approval Institutional review board approval was obtained.

Study subjects or cohorts overlap 10 study subjects have been previously reported in PMID: 23525206.

\author{
Methodology \\ - retrospective \\ - observational \\ - performed at one institution
}

Open Access This article is distributed under the terms of the Creative Commons Attribution 4.0 International License (http:// creativecommons.org/licenses/by/4.0/), which permits unrestricted use, distribution, and reproduction in any medium, provided you give appropriate credit to the original author(s) and the source, provide a link to the Creative Commons license, and indicate if changes were made.

\section{References}

1. Zietman AL, Bae K, Slater JD et al (2010) Randomized trial comparing conventional-dose with high-dose conformal radiation therapy in early-stage adenocarcinoma of the prostate: long-term results from Proton Radiation Oncology Group/American College Of Radiology 95-09. J Clin Oncol 28:1106-1111

2. Suzuki N, Shimbo M, Amiya Y et al (2010) Outcome of patients with localized prostate cancer treated by radiotherapy after confirming the absence of lymph node invasion. Jpn J Clin Oncol 40:652-657

3. Agarwal PK, Sadetsky N, Konety BR et al (2008) Treatment failure after primary and salvage therapy for prostate cancer: likelihood, patterns of care, and outcomes. Cancer 112:307-314 
4. Cornford P, Bellmunt J, Bolla M et al (2017) EAU-ESTRO-SIOG guidelines on prostate cancer. Part II: treatment of relapsing, metastatic, and castration-resistant prostate cancer. Eur Urol 71:630-642

5. Gotto GT, Yunis LH, Vora K et al (2010) Impact of prior prostate radiation on complications after radical prostatectomy. J Urol 184: 136-142

6. Finley DS, Belldegrun AS (2011) Salvage cryotherapy for radiation-recurrent prostate cancer: outcomes and complications. Curr Urol Rep 12:209-215

7. Wenske S, Quarrier S, Katz AE (2013) Salvage cryosurgery of the prostate for failure after primary radiotherapy or cryosurgery: longterm clinical, functional, and oncologic outcomes in a large cohort at a tertiary referral centre. Eur Urol 64:1-7

8. Babaian RJ, Donnelly B, Bahn D et al (2008) Best practice statement on cryosurgery for the treatment of localized prostate cancer. J Urol 180:1993-2004

9. De Castro Abreu AL, Bahn D, Leslie S et al (2013) Salvage focal and salvage total cryoablation for locally recurrent prostate cancer after primary radiation therapy. BJU Int 112:298-307

10. Eisenberg ML, Shinohara K (2008) Partial salvage cryoablation of the prostate for recurrent prostate cancer after radiotherapy failure. Urology 72:1315-1318

11. Li Y-H, Elshafei A, Agarwal G et al (2015) Salvage focal prostate cryoablation for locally recurrent prostate cancer after radiotherapy: Initial results from the cryo on-line data registry. Prostate 75:1-7

12. Yakar D, Hambrock T, Huisman H et al (2010) Feasibility of $3 T$ dynamic contrast-enhanced magnetic resonance-guided biopsy in localizing local recurrence of prostate cancer after external beam radiation therapy. Investig Radiol 45:121-125

13. Barchetti F, Panebianco V (2014) Multiparametric MRI for recurrent prostate cancer post radical prostatectomy and postradiation therapy. Biomed Res Int. doi:10.1155/2014/316272

14. Morrison PR, Silverman SG, Tuncali K, Tatli S (2008) MRI-guided cryotherapy. J Magn Reson Imaging 27:410-420

15. Gangi A, Tsoumakidou G, Abdelli O et al (2012) Percutaneous MR-guided cryoablation of prostate cancer: initial experience. Eur Radiol 22:1829-1835

16. Bomers JGR, Yakar D, Overduin CG et al (2013) MR imagingguided focal cryoablation in patients with recurrent prostate cancer. Radiology 268:451-460

17. Baust J, Gage AA, Ma H, Zhang CM (1997) Minimally invasive cryosurgery - technological advances. Cryobiology 34:373-384

18. Young JL, Kolla SB, Pick DL et al (2010) In vitro, ex vivo and in vivo isotherms for renal cryotherapy. J Urol 183:752-758

19. Lee F, Bahn DK, McHugh TA et al (1994) US-guided percutaneous cryoablation of prostate cancer. Radiology 192:769-776

20. Mohler J, Bahnson RR, Boston B et al (2010) Prostate cancer. Natl Compr Cancer Netw 8:162-200

21. Overduin CG, Bomers JGR, Jenniskens SFM et al (2014) T1weighted MR image contrast around a cryoablation iceball: a phantom study and initial comparison with in vivo findings. Med Phys 41:112301

22. Woodrum DA, Kawashima A, Karnes RJ et al (2013) Magnetic resonance imaging-guided cryoablation of recurrent prostate cancer after radical prostatectomy: initial single institution experience. Urology 82:870-875

23. Roach M, Hanks G, Thames H et al (2006) Defining biochemical failure following radiotherapy with or without hormonal therapy in men with clinically localized prostate cancer: recommendations of the RTOG-ASTRO Phoenix Consensus Conference. Int J Radiat Oncol Biol Phys 65:965-974

24. Ahmed HU, Pendse D, Illing R et al (2007) Will focal therapy become a standard of care for men with localized prostate cancer? Nat Clin Pract Oncol 4:632-642
25. Baust JG, Gage AA (2005) The molecular basis of cryosurgery. BJU Int 95:1187-1191

26. Littrup PJ, Jallad B, Vorugu V et al (2009) Lethal isotherms of cryoablation in a phantom study: effects of heat load, probe size, and number. J Vasc Interv Radiol 20:1343-1351

27. Georgiades C, Rodriguez R, Azene E et al (2013) Determination of the nonlethal margin inside the visible "ice-ball" during percutaneous cryoablation of renal tissue. Cardiovasc Intervent Radiol 36: 783-790

28. Ge BH, Guzzo TJ, Nadolski GJ et al (2016) Percutaneous renal cryoablation: short-axis ice-ball margin as a predictor of outcome. J Vasc Interv Radiol 27:403-409

29. Mala T, Samset E, Aurdal L et al (2001) Magnetic resonance imaging-estimated three-dimensional temperature distribution in liver cryolesions: a study of cryolesion characteristics assumed necessary for tumor ablation. Cryobiology 43:268-275

30. Young JL, McCormick DW, Kolla SB et al (2012) Are multiple cryoprobes additive or synergistic in renal cryotherapy? Urology 79:484.e1-484.e6

31. Le Nobin J, Orczyk C, Deng FM et al (2014) Prostate tumour volumes: Evaluation of the agreement between magnetic resonance imaging and histology using novel co-registration software. BJU Int 114:E105-E112

32. Le Nobin J, Rosenkrantz AB, Villers A et al (2015) Image guided focal therapy for magnetic resonance imaging visible prostate cancer: defining a 3-dimensional treatment margin based on magnetic resonance imaging histology co-registration analysis. J Urol 194: 364-370

33. Groenendaal G, Moman MR, Korporaal JG et al (2010) Validation of functional imaging with pathology for tumor delineation in the prostate. Radiother Oncol 94:145-150

34. Bolla M, Collette L, Blank L et al (2002) Long-term results with immediate androgen suppression and external irradiation in patients with locally advanced prostate cancer (an EORTC study): a phase III randomised trial. Lancet 360:103-108

35. D'Amico AV, Manola J, Loffredo M et al (2004) 6-month androgen suppression plus radiation therapy vs radiation therapy alone for patients with clinically localized prostate cancer: a randomized controlled trial. JAMA 292:821-827

36. Garzotto M, Wajsman Z (1998) Androgen deprivation with salvage surgery for radiorecurrent prostate cancer: results at 5-year followup. J Urol 159:950-4-5

37. Li R, Ruckle HC, Schlaifer AE et al (2015) The effect of androgen deprivation therapy before salvage whole-gland cryoablation after primary radiation failure in prostate cancer treatment. Urology 85 : $1137-1142$

38. Fortuin AS, Smeenk RJ, Meijer HJM et al (2014) Lymphotropic nanoparticle-enhanced MRI in prostate cancer: Value and therapeutic potential. Curr Urol Rep. doi:10.1007/s11934-013-0389-7

39. Birkhäuser FD, Studer UE, Froehlich JM et al (2013) Combined ultrasmall superparamagnetic particles of iron oxide-enhanced and diffusion-weighted magnetic resonance imaging facilitates detection of metastases in normal-sized pelvic lymph nodes of patients with bladder and prostate cancer. Eur Urol 64:953-960

40. Eiber M, Maurer T, Souvatzoglou M et al (2015) Evaluation of hybrid 68Ga-PSMA ligand PET/CT in 248 patients with biochemical recurrence after radical prostatectomy. J Nucl Med 56:668-674

41. Pitman M, Shapiro EY, Hruby GW et al (2012) Comparison of biochemical failure definitions for predicting local cancer recurrence following cryoablation of the prostate. Prostate 72:18021808

42. De Visschere PJ, De Meerleer GO, Fütterer JJ, Villeirs GM (2010) Role of MRI in follow-up after focal therapy for prostate carcinoma. Am J Roentgenol 194:1427-1433 